\title{
Nanostructure design for surface-enhanced Raman spectroscopy - prospects and limits
}

\section{Sanshui Xiao \\ Niels Asger Mortensen asger@mailaps.org}

\section{Antti-Pekka Jauho}

\author{
Department of Photonics Engineering, Technical University of Denmark, DTU Fotonik, DTU-building \\ 345 west, DK-2800 Kongens Lyngby \\ Department of Micro and Nanotechnology, Technical University of Denmark, DTU Nanotech, DTU- \\ building 345 east, DK-2800 Kongens Lyngby, Denmark \\ Laboratory of Physics, Helsinki University of Technology, P. O. Box 1100, FI-02015 HUT, Finland
}

Surface-enhanced Raman spectroscopy (SERS) allows single-molecule detection due to the strong field localization occurring at sharp bends or kinks of the metal-vacuum interface. An important question concerns the limits of the signal enhancement that can be achieved via a judicious design of the surface. By using a specific example of a technologically realizable nanopatterned surface, we demonstrate that while very high enhancement factors $\left(\approx 10^{12}\right.$ ) can be found for an ideal surface, these are unlikely to be achieved in laboratory samples, because even a minute, inevitable rounding-off strongly suppresses the enhancement, as well as shifts the optimal frequency. Our simulations indicate that the geometric enhancement factors are unlikely to exceed $\approx 10^{8}$ for real samples, and that it is necessary to consider the geometric uncertainty to reliably predict the frequency for maximum enhancement. [D0I: 10.2971/je0s.2008.08022]

Keywords: plasmonics, SERS

\section{INTRODUCTION}

Raman spectroscopy is a powerful technique which has become one of the optical workhorses in analytical chemistry where it is used to detect and quantify a variety of chemical substances including biomolecules absorbed to a substrate. The 1974 report by Fleischmann et al. [1] on surface-enhanced Raman spectroscopy (SERS) created an avalanche in surfaceenhanced spectroscopies [2]. Localized surface plasmons may dramatically enhance the otherwise intrinsically weak Raman scattering [see panel (a) in Figure 1] and this has paved the way for chemical detection in very dilute solutions, reaching the limit of single-molecule detection [3]. Initially, there was a strong emphasis on disordered and rough metal surfaces [4]; however during the past decade the use of highly engineered surface plasmonic structures has been envisioned [5]. The latter development has to a large extent been fueled by the rapid development of a variety of nanofabrication technologies, including high-resolution electron-beam lithography on the true nano scale. As a consequence, surface plasmon subwavelength optics has in general developed tremendously [6], and in particular SERS has been observed for a large variety of nanostructures, such as nanorods, nanorings, triangular nanoprisms, nanocubes, nanocrescents, and nanorice [7].

Field localization is the key to understanding and further enhancing SERS. Structures with pronounced bends in their surface $\partial \mathscr{V}$ will tend to localize the field also in the plane of the surface [8]. Singular points, such as sharp kinks and corners, may lead to nonanalytic or even mathematically divergent electric fields. Real structures are smooth, of course, at least on some microscopic length scale. All fabrication processes have their inherent upper bounds on the sharpness that can be fabricated reproducibly. As an example, state-of-the-art groove waveguides have sharp features down to a $10 \mathrm{~nm}$ scale [9]. In an ensemble of nanostructures there will always be a size dispersion or structure-to-structure fluctuations in some de-
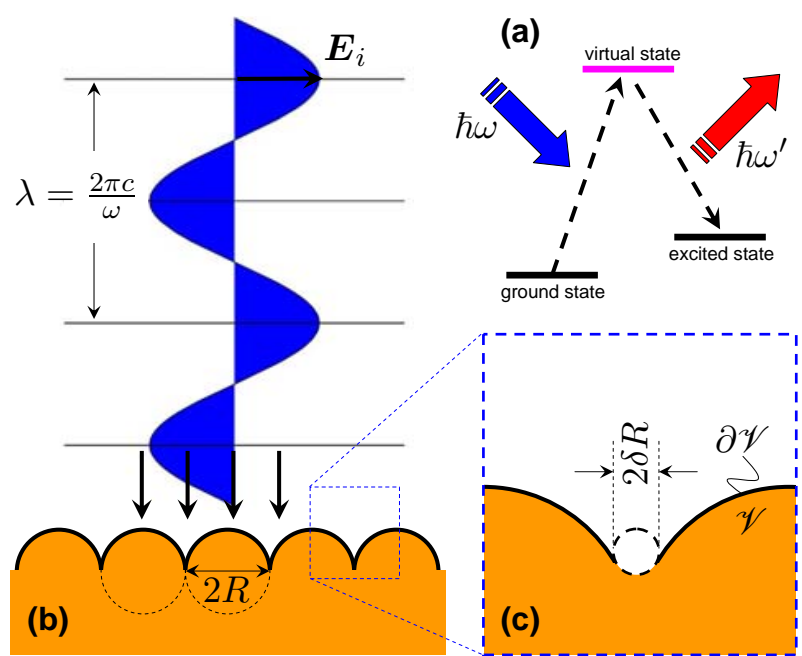

FIG. 1 (color online) (a) Energy diagram for the Raman scattering process involving the absorption of an incident photon with energy $\hbar \omega$, and a subsequent emission of a photon with energy $\hbar \omega^{\prime}$ via an intermediate virtual state in a molecule. The scattering is inelastic and the scattered light is Stokes shifted by $\Delta \omega=\omega-\omega^{\prime}$. (b) Metallic periodic structure composed of infinite half-cylinders of radius $R$. The normal incident electrical field $\mathbf{E}_{i}$ is polarized transverse to the cylinders. (c) Magnification of the groove structure indicating the nanoscale cutoff modelled by a finite radius of curvature $\delta R$. 
tails of the geometry, for example the nanoscale rounding $\delta R$ [10] will differ from structure to structure, with possibly far-reaching effects on the SERS. Fluctuations between repeated features and variations in the smoothness of individual features have previously been addressed for fractal structures in a number of papers by Sánchez-Gil et al., see e.g. $[12,13]$. The pronounced sample-to-sample fluctuations are potentially a serious challenge to the development of a technology for reliable use in quantitative sensing applications and bio-chemical analysis.

In this paper we address another crucial issue: how are the high enhancement factors found for highly ideal surface structures affected by round-off effects? By means of numerical examples we shall illustrate the extreme precision needed in the fabrication of nanostructured surfaces, if one aims at SERS in the excess of ten orders of magnitude. Specifically, we consider the simple model geometry in panel (b) of Figure 1. Historically, this was the first structure considered theoretically to explain the large enhancement occurring for rough metal surfaces [4]. Today, we may easily envision realization of groove structures [14] closely resembling the geometry considered in panel (c), though state-of-the-art structures may still be somewhat larger in scale. As mentioned above, the points of intersection of neighboring cylinders may lead to a singularity in the electric field, and consequently arbitrarily large enhancement factors [see Eq. (1) below].

\section{THEORY}

In the context of SERS it is customary to estimate the surfaceenhancement factor $\gamma$ from [4]

$$
\gamma(\omega) \equiv \frac{\int_{\partial \mathscr{V}} d \mathbf{r} \gamma(\mathbf{r}, \omega)}{\int_{\partial \mathscr{V}} d \mathbf{r}}, \gamma(\mathbf{r}, \omega) \approx \frac{\left|\mathbf{E}_{t}(\mathbf{r}, \omega)\right|^{4}}{\left|\mathbf{E}_{i}(\mathbf{r}, \omega)\right|^{4}},
$$

where $\mathbf{r}$ is the position of the molecule. To a first approximation the surface-enhancement factor is independent on the intrinsic properties of the molecule. Here, $\partial \mathscr{V}$ is the surface bounding the volume $\mathscr{V}$ occupied by metal, $\mathbf{E}_{i}$ is the incident field, and the total field is $\mathbf{E}_{t}=\mathbf{E}_{i}+\mathbf{E}_{s}$ with $\mathbf{E}_{s}$ being the scattered component that arises in the presence of the metal surface. The surface-averaged enhancement factor applies to the case of a spatially uniform surface-coverage of molecules.

Our theoretical study takes the wave equation for the electrical field as a starting point

$$
\nabla \times \nabla \times \mathbf{E}(\mathbf{r})=\frac{\omega^{2}}{c^{2}} \varepsilon(\mathbf{r}, \omega) \mathbf{E}(\mathbf{r})
$$

where $\omega$ is the angular frequency and $c$ is the vacuum velocity of light. For structures as in Figure 1 the spatially dependent relative dielectric function is of the form

$$
\varepsilon(\mathbf{r}, \omega)=\left\{\begin{array}{cc}
1, & \mathbf{r} \notin \mathscr{V} \\
\varepsilon(\omega), & \mathbf{r} \in \mathscr{V}
\end{array}\right.
$$

where $\varepsilon(\omega)$ is the dielectric function of the metal. To make our simulations mimic a real material we use the bulk dielectric constant of silver (thus neglecting spatial dispersion near the metal surface), illustrated in Figure 2 which shows standard handbook data for $\varepsilon(\omega)$ [15] along with a numerical fit to the Drude expression

$$
\varepsilon(\omega)=\varepsilon_{\infty}+\frac{\left(\varepsilon_{0}-\varepsilon_{\infty}\right) \omega_{p}^{2}}{\omega^{2}+i \omega / \tau}
$$

with $\omega_{p}$ being the plasma frequency and $\tau^{-1}$ the damping rate. This simple model accounts very well for the real part, however some deviations are seen in the visible for the imaginary part. The spectral position of resonances will thus be accurately predicted by using the Drude fit while we shall see that even small deviations in the imaginary part of $\varepsilon$ may influence the enhancement factor, Eq. (1), by orders of magnitude.

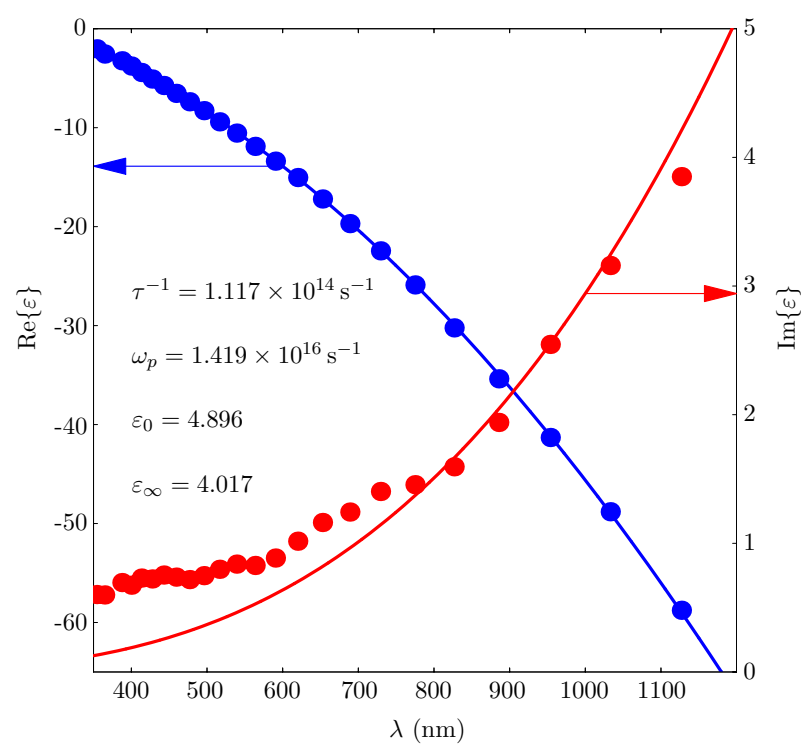

FIG. 2 (color online) Dielectric function of silver. The data points show handbook data from Ref. [15] while the solid lines are fits to the Drude model in Eq. (4).

\section{NUMERICAL RESULTS}

In the following we use a finite-element approach (Comsol Multiphysics) to solve Eq. (2) in a unit cell resembling panel (c) in Figure 1. We apply periodic boundary conditions in the direction parallel to the surface. Deep inside the metal and far out in the free space we employ perfectly-matched layers in the perpendicular direction [16], so that the domain has an extension $\gg \lambda$, i.e. significantly exceeding the free-space wavelength. Eq. (2) is solved for a normal incident electrical field polarized perpendicular to the groove axis, see panel (b) in Figure 1, which strongly polarizes the surface by excitation of a surface-plasmon resonance. With the resulting scattered field, and hence $E_{t}$, we subsequently evaluate Eq. (1) by a boundary integration routine. Our meshing is chosen to ensure convergence of the enhancement factor; this requires a particularly fine mesh in the groove region. As an example, resolving the peak in $\gamma$ at resonance has typically required a sub-nanometer meshing. On a standard-equipped PC the calculation of a single near-resonance frequency point typically takes around 30 seconds.

Figure 3 shows the wavelength dependence of the surfaceaveraged enhancement factor in the case of $R=15 \mathrm{~nm}$ and 


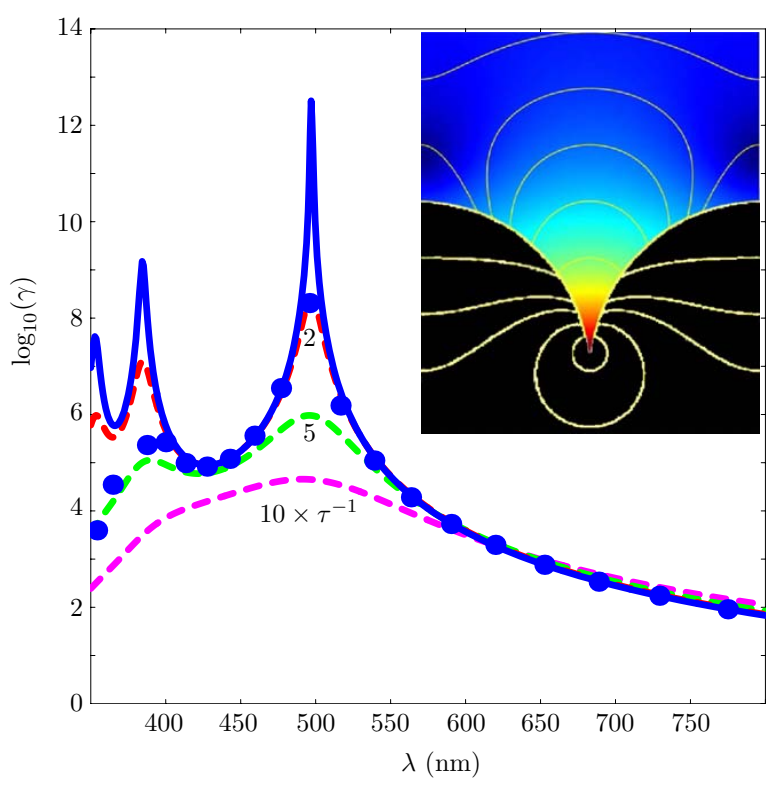

FIG. 3 (color online) Wavelength dependence of the surface-averaged enhancement factor for a structure with $R=15 \mathrm{~nm}$ and $\delta R=0.1 \mathrm{~nm}$. The data points result from direct simulations at discrete frequencies using handbook data for silver, see Figure 2 , while the solid line shows the corresponding results based on the Drude model, Eq. (4). The dashed lines illustrate the suppression of the SERS effect for increasing damping rates $\tau^{-1}$. The inset shows $\gamma$ at the main peak where the electrical field is strongly localized in the groove. The electrical field points in a direction parallel to the superimposed electric field lines.

$\delta R=0.1 \mathrm{~nm}$. The solid line shows results based on the Drude model, Eq. (4), while the points are obtained from a simulation using the discrete frequencies where handbook data is available, see Figure 2. First, we note that these geometrical parameters easily lead to surface-averaged enhancement factors in excess of $10^{12}$, i.e. twelve orders of magnitude, in the visible. Second, when inspecting the peak around $\lambda \sim 500 \mathrm{~nm}$ and comparing the two simulations, it is also clear that the Drude model, Eq. (4), overestimates the enhancement by perhaps up to three orders of magnitude. This overestimation may be traced back to the imaginary part of $\varepsilon$ shown in Figure 2 where the Drude model seriously underestimates the broadening of the surface-plasmon resonance. Finally, when approaching the ultra-violet, resonances predicted by the Drude model are barely visible when employing the handbook data directly. While general trends can be extracted from a Drude description, these results emphasize the need for an accurate material-dispersion law for even semi-quantitative predictions of SERS. In particular, the surface-plasmon damping is crucial in the vicinity of resonances as clearly seen in the dashed traces where we for illustrative purposes have increased the damping rate $\tau^{-1}$ by a factor of two, five, and ten.

While previous studies [4] have reported a spectrally broadened enhancement, our detailed finite-element simulations suggest the existence of highly peaked enhancement factors associated with the formation of a surface-plasmon resonance strongly localized in the groove. A related transverse localization has recently been reported experimentally for groove waveguides [14] and subsequently confirmed numerically [8]. Obviously, spatial localization is the route to high enhancement and especially one should minimize the overlap with the lossy metal causing Ohmic broadening of resonances. The nanoscale cutoff $\delta R$ is the geometrical knob that controls the strength of localization and the overlap with the metal. A stronger localization ( $\delta R \rightarrow 0$ ) will usually be expected to squeeze up the resonance in frequency space. However, when increasing $\delta R$ we actually observe a blue-shift which can be understood within the quasi-static framework of [17] [see their Eq. (4)] where an increase in metal volume $\mathscr{V}$ causes modes at $\omega$ to blue-shift to a frequency $\omega+\Delta \omega$ with a less negative dielectric function, i.e. $\varepsilon(\omega+\Delta \omega)>\varepsilon(\omega)$. In Figure 4 we illustrate this point for varying values of $\delta R$ at fixed $R=15 \mathrm{~nm}$. For simplicity the results are based on the Drude model. Our results show that even for a relatively conservative value of the ratio $\delta R / R \sim 3 \%$ the enhancement has already dropped to $\gamma \sim 10^{8}$ compared to $\gamma \sim 10^{12}$ in the case of $\delta R / R \lesssim 1 \%$.

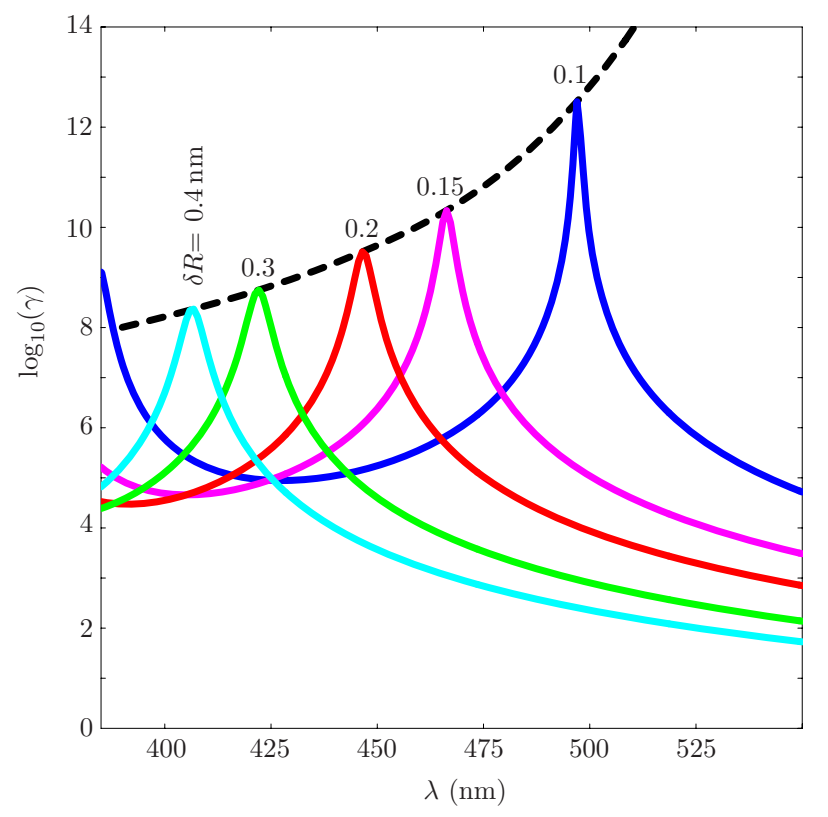

FIG. 4 (color online) Wavelength dependence of the surface-averaged enhancement factor for a structure with $R=15 \mathrm{~nm}$ and varying nanoscale cutoff $\delta R$.

Another geometric factor affecting the field enhancement is the radius of cylinders. In Figure 5 we illustrate this point for varying values of $R$ at fixed $\delta R=1 \mathrm{~nm}$, again using the Drude model. Our results show a red-shift of the resonance when increasing the radius while the magnitude decreases slightly. The red-shift may be understood qualitatively from the scale invariance of Eq. (2) in the absence of material dispersion. Figures 4 and 5 together illustrate how the enhancement is influenced by both length scales $R$ and $\delta R$ in a nontrivial interplay.

\section{CONCLUSION}

In conclusion, we have presented finite-element simulations of the SERS enhancement factor for an example of a nanostructured surface, which represents the state-of-the-art technology. We find that a well-defined structure, with mathematically sharp boundaries [see Eq. (3)], may exhibit huge enhancement factors, $\gamma \approx 10^{12}$. But any geometric smoothening rapidly decreases the enhancement, and even though a computational optimization may be possible by a painstaking survey of the parameter space (given that the dielectric function of 


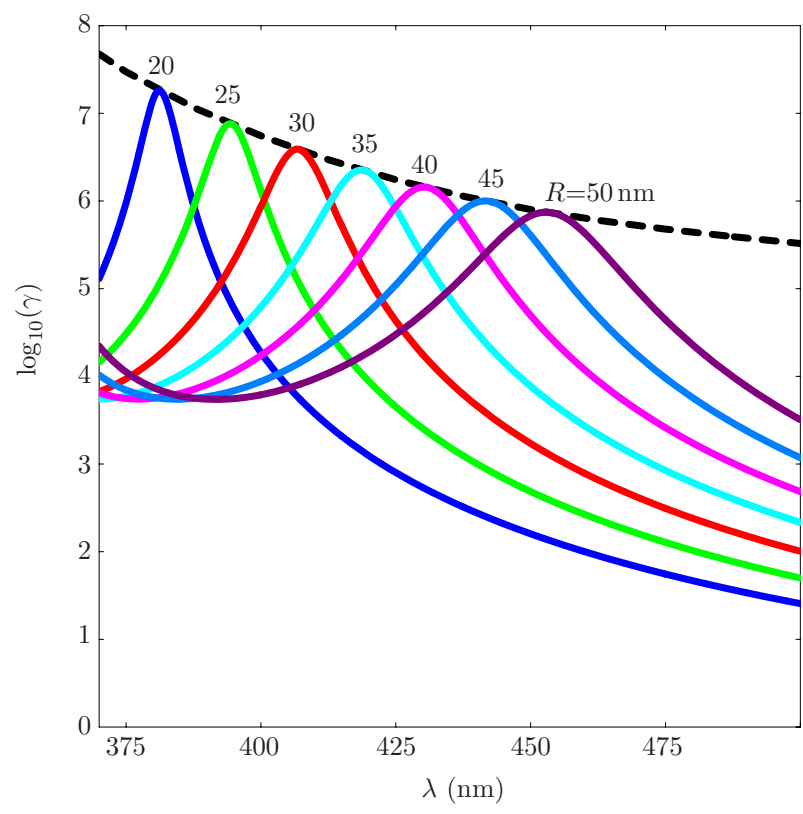

FIG. 5 (color online) Wavelength dependence of the surface-averaged enhancement factor for a structures with varying radius $R$ of the semi cylinders and a nanoscale cutoff $\delta R=1 \mathrm{~nm}$.

the material is known very accurately - no small feat by itself!), it is doubtful whether the extremely high theoretical enhancement factors can be reached in practice and in a reproducible manner.

\section{ACKNOWLEDGEMENTS}

We acknowledge stimulating discussions with S. I. Bozhevolnyi. This work is financially supported by the Danish Council for Strategic Research through the Strategic Program for Young Researchers (grant no: 2117-05-0037) and the Danish Research Council for Technology and Production Sciences (grant no: 274-070379) as well as the FiDiPro program of the Finnish Academy.

\section{References}

[1] M. Fleischmann, P. J. Hendra, and A. J. McQuillan, "Raman-spectra of pyridine adsorbed at a silver electrode" Chem. Phys. Lett. 26, 163-166 (1974).

[2] M. Moskovits, "Surface-enhanced spectroscopy" Rev. Mod. Phys.
57, $783-826$ (1985).

[3] K. Kneipp, Y. Wang, H. Kneipp, L. T. Perelman, I. Itzkan, R. Dasari, and M. S. Feld, "Single molecule detection using surface-enhanced Raman scattering (SERS)" Phys. Rev. Lett. 78, 1667 - 1670 (1997).

[4] F. J. García-Vidal, and J. B. Pendry, "Collective theory for surface enhanced Raman scattering" Phys. Rev. Lett. 77, 1163- 1166 (1996).

[5] J. B. Pendry, L. Martín-Moreno, and F. J. García-Vidal, “Mimicking surface plasmons with structured surfaces" Science $305,847-848$ (2004).

[6] W. L. Barnes, A. Dereux, and T. W. Ebbesen, "Surface plasmon subwavelength optics" Nature 424, 824 - 830 (2003).

[7] S. Lal, S. Link, and N. J. Halas, "Nano-optics from sensing to waveguiding" Nature Photonics 1, $641-648$ (2007).

[8] E. Moreno, F. J. García-Vidal, S. G. Rodrigo, L. Martín-Moreno, and S. I. Bozhevolnyi, "Channel plasmon-polaritons: modal shape, dispersion, and losses" Opt. Lett. 31, $3447-3449$ (2006).

[9] S. I. Bozhevolnyi, (private communication).

[10] A similar model has been used in the context of groove waveguide structures [8] and square-rod metal waveguides [11].

[11] J. Jung, T. Søndergaard, and S. I. Bozhevolnyi, "Theoretical analysis of square surface plasmon-polariton waveguides for long-range polarization-independent waveguiding" Phys. Rev. B 76, 035434 (2007).

[12] J. A. Sánchez-Cil, J. V. García-Ramos, and E. R. Méndez, “Near-field electromagnetic wave scattering from random self-affine fractal metal surfaces: Spectral dependence of local field enhancements and their statistics in connection with surface-enhanced Raman scattering" Phys. Rev. B 62, 10515 - 10525 (2000).

[13] J. A. Sánchez-Gil, J. V. García-Ramos, and E. R. Méndez, “Electromagnetic mechanism in surface-enhanced Raman scattering from Gaussian-correlated randomly rough metal substrates" opt. Express 10, $879-886$ (2002).

[14] S. I. Bozhevolnyi, V. S. Volkov, E. Devaux, J. Y. Laluet, and T. W. Ebbesen, "Channel plasmon subwavelength waveguide components including interferometers and ring resonators" Nature 440, $508-511$ (2006).

[15] E. D. Palik, Handbook of Optical Constants of Solids (Academic, New York, 1985).

[16] J. P. Berenger, "A perfectly matched layer for the absorption of electromagnetic waves" J. Comput. Phys. 114, 185-200 (1994).

[17] F. Wang, and Y. R. Shen, "General properties of local plasmons in metal nanostructures" Phys. Rev. Lett. 97, 206806 (2006). 